\title{
Pomeron structure functions from HERA to Tevatron and LHC
}

\author{
C. Royon*, L. Schoeffel ${ }^{\dagger}$, R.Peschanski ${ }^{\ddagger}$, E.Sauvan ${ }^{\S}$
}

\begin{abstract}
The proton diffractive structure function $F_{2}^{D(3)}$ measured in the $\mathrm{H} 1$ and ZEUS experiments at HERA are analysed in terms of perturbative QCD in the perspective of the QCD extrapolation to the Tevatron and the LHC. It is shown that both data sets can be well described by a QCD analysis in which point-like parton distributions, evolving according to the next-leading DGLAP equations, are assigned to the leading and sub-leading Regge exchanges. For present data from H1 and ZEUS the gluon distributions are found to be quite different and we give the corresponding sets of quark and gluon parton distributions for the Pomeron, extracted from the two experiments. An extrapolation to the Tevatron range is compared with CDF data on single diffraction. Conclusions on factorization breaking between HERA and Tevatron critically depend on whether H1 (strong violation) or ZEUS (compatibility at low $\beta$ ) fits are taken into account. Using the double Pomeron formulation in central diffractive dijet production we show that the Tevatron mass fraction is much sensitive to the high $\beta$ tail of the gluon in the Pomeron, suggesting a new way of handling the otherwise badly known gluon distribution in the Pomeron. Extrapolation of the fits to very high $Q^{2}$ are given since they will be relevant for QCD and diffraction studies at the LHC.
\end{abstract}

\section{INTRODUCTION}

Since years, the Pomeron remains a subject of many interrogations. Indeed, defined as the virtual colourless carrier of strong interactions, the nature of the Pomeron is still a real challenge. While in the perturbative regime of QCD it can be defined as a compound system of two perturbatively Reggeized gluons [1] in the approximation of resumming the leading logs in energy, its non-perturbative structure is basically unknown.

However, in the recent years, an interesting experimental investigation on "hard" diffractive processes led to a new insight into Pomeron problems. It is now experimentally well established at HERA [2-4] that a substantial fraction of $e p$ events is contributable to diffraction, i.e. color singlet exchange, initiated by a highly virtual photon. Starting with the pioneering theoretical work of Ref. [5], the idea of a point-like structure of the Pomeron exchange opens the way to the determination of its parton (quark and gluon) distributions, where the Pomeron point-like structure can be treated in a similar way as (and compared to) the proton one. Indeed, leading twist contributions to the proton diffractive structure functions can be defined by factorization properties [6] in much the same way as for the full proton structure functions themselves. As such, they should obey DGLAP evolution equations [7], and thus allow for perturbative predictions of their $Q^{2}$ evolution.

In Ref. [8], we have shown that the data are well described by a QCD analysisin which point-like parton distributions, evolving according to the DGLAP equations, are assigned to the leading and sub-leading Regge exchanges. The gluon distributions were found to be quite different for H1 and ZEUS. Then, in Ref. [8], we have derived sets of quark and gluon parton distributions for the Pomeron, and predictions for the charm and the longitudinal diffractive structure function from the QCD fit. An extrapolation to the Tevatron range was also compared with CDF data on single diffraction. In Ref. [9], we have asked the question whether the quark and gluon distributions in the Pomeron obtained from QCD fits to hard diffraction processes at HERA could be dynamically generated by QCD evolution from a state made of valence-like gluons and sea quarks as an input. By a method combining backward $Q^{2}$-evolution for data exploration and forward $Q^{2}$-evolution for a best fit determination, we have found that the diffractive structure functions published by the H1 collaboration at HERA could be described by a simple valence-like input at an initial low scale. The same property was not achieved when using ZEUS data.

These previous analyses were based on diffractive deep inelastic scattering (DIS) cross-sections determined at HERA $[2,3]$. It can be seen as a first stage in studying aspects of the QCD fit technique applied to diffraction, as for example the flexibility of the parton distributions parameterisations.

In the present paper, we produce new sets of diffractive parton distribution functions (DPDFs) extracted from the presently published data sets, which consist in the already mentionned set for H1 [2] and the latest results from ZEUS

\footnotetext{
*Service de Physique des Particules, CE-Saclay, F-91191 Gif-sur-Yvette Cedex, France

${ }^{\dagger}$ Service de Physique des Particules, CE-Saclay, F-91191 Gif-sur-Yvette Cedex, France

${ }^{\ddagger}$ Service de Physique Théorique, Unité de recherche associée au CNRS, CE-Saclay, F-91191 Gif-sur-Yvette Cedex, France

${ }^{\S}$ CPPM, F-13288 Marseille Cedex 09, France Marseille, France
} 
diffractive DIS cross-sections [4]. The results on DPDFs are close to those of Ref. [8]. We give for the first time a new method to evaluate the uncertainty on the gluon density at large values of $\beta$, which is a key point for any further discussion of the influence of DPDFs at Tevatron or LHC.

Then, we present the main result of this article, which is to show how these diffractive parton densities enter in the simulations of the dijet mass fraction at the Tevatron in central diffractive dijet production within the double Pomeron formulation. We show that this quantity is much sensitive to the high $\beta$ tail of the gluon in the Pomeron. Our analysis allows to provide the extrapolation of the fits to very high $Q^{2}$ since they will be relevant for QCD and hard diffraction studies at the LHC.

\section{EXTRACTION OF PARTON DISTRIBUTIONS IN THE POMERON AND QCD FITS}

\section{A. Formulation}

It is well known $[2,8]$ that the diffractive structure function $F_{2}^{D(3)}$, measured from DIS events with large rapidity gaps, can be investigated in the framework of Regge factorization and expressed as a sum of two factorized contributions corresponding to a Pomeron and secondary Reggeon trajectories :

$$
F_{2}^{D(3)}\left(Q^{2}, \beta, x_{\mathbb{P}}\right)=f_{\mathbb{P} / \mathrm{p}}\left(x_{\mathbb{P}}\right) F_{2}^{\mathbb{P}}\left(Q^{2}, \beta\right)+f_{\mathbb{R} / \mathrm{p}}\left(x_{\mathbb{P}}\right) F_{2}^{\mathbb{R}}\left(Q^{2}, \beta\right) .
$$

In this parameterisation, $F_{2}^{\mathbb{P}}$ can be interpreted as the Pomeron structure function and $F_{2}^{\mathbb{R}}$ as an effective Reggeon structure function, with the restriction that it takes together into account various secondary Regge contributions which can hardly be separated. The Pomeron, $f_{\mathbb{P} / \mathrm{p}}$, and Reggeon, $f_{\mathbb{R} / \mathrm{p}}$, fluxes are assumed to follow a Regge behaviour with linear trajectories $\alpha_{\mathbb{P}, \mathbb{R}}(t)=\alpha_{\mathbb{P}, \mathbb{R}}(0)+\alpha_{\mathbb{P}, \mathbb{R}}^{\prime} t$, such that

$$
f_{\mathbb{P} / p, \mathbb{R} / p}\left(x_{\mathbb{P}}\right)=\int_{t_{c u t}}^{t_{m i n}} \mathrm{~d} t \frac{e^{B_{\mathbb{P}, \mathbb{R}} t}}{x_{\mathbb{P}}^{2 \alpha_{\mathbb{P}, \mathbb{R}}(t)-1}}
$$

where $\left|t_{m i n}\right|$ is the minimal kinematically-allowed value of $|t|$ and $t_{c u t}=-1 \mathrm{GeV}^{2}$ is the limit of the measurement. The values of the $t$-slope parameters are taken from hadron-hadron data $\left(\alpha_{\mathbb{P}}^{\prime}=0.26 \mathrm{GeV}^{-2}, \alpha_{\mathbb{R}}^{\prime}=0.90 \mathrm{GeV}^{-2}\right.$, $B_{\mathbb{P}}=4.6 \mathrm{GeV}^{-2}, B_{\mathbb{R}}=2.0 \mathrm{GeV}^{-2}$ ), since the data are not precise enough to determine them [2].

We assign parton distribution functions to the Pomeron and to the Reggeon, considered as coumpound states of fundamental quarks and gluons. A simple and well-known prescription of QCD factorisation is that the parton distributions of both the Pomeron and the Reggeon are parameterized in terms of non-perturbative input distributions at some low scale which was adopted here to be $Q_{0}^{2}=3 \mathrm{GeV}^{2}$. The pion structure function [13] is assumed to be also valid for the sub-leading Reggeon trajectory with a free global normalization to be determined by the data ${ }^{1}$.

For the Pomeron, a quark flavour singlet distribution, $z S\left(z, Q^{2}\right)=u+\bar{u}+d+\bar{d}+s+\bar{s}$, and a gluon distribution, $z G\left(z, Q^{2}\right)$, are parameterized in termsof coefficients $C_{j}^{(S)}$ and $C_{j}^{(G)}$ at $Q_{0}^{2}=3 \mathrm{GeV}^{2}$ as it was done in Ref. [2] such that

$$
\begin{aligned}
& z S\left(z, Q^{2}=Q_{0}^{2}\right)=\left(\sum_{j=1}^{n} C_{j}^{(S)} \cdot P_{j}(2 z-1)\right)^{2} \cdot \exp \left(\frac{a}{z-1}\right) \\
& z G\left(z, Q^{2}=Q_{0}^{2}\right)=\left(\sum_{j=1}^{n} C_{j}^{(G)} \cdot P_{j}(2 z-1)\right)^{2} \cdot \exp \left(\frac{a}{z-1}\right)
\end{aligned}
$$

where $z=x_{i / \mathbb{P}}$ is the fractional momentum of the Pomeron carried by the struck parton, $P_{j}(\zeta)$ is the $j^{\text {th }}$ member in a set of Chebyshev polynomials, chosen such that $P_{1}=1, P_{2}=\zeta$, and $P_{j+1}(\zeta)=2 \zeta P_{j}(\zeta)-P_{j-1}(\zeta)$. In the following,

\footnotetext{
${ }^{1}$ We checked that changing the pion structure function by some amount (20\%) does not change significantly the parton distributions. For even better checking, another pion structure function [14] has also been used without any sizable modification of the results.
} 
we present the results in terms of the overall quark density with the hypothesis that $u=\bar{u}=d=\bar{d}=s=\bar{s}$, which means that each light quark density is equal to $z S / 6$.

A sum of $n=3$ orthonormal polynomials is used so that the input distributions are free to adopt a large range of forms for a given number of parameters. Any bias towards a particular solution due to the choice of the functional form of the input distribution is therefore minimized.

In Ref. [8], we have used the following fixed Pomeron intercepts for H1 and ZEUS data sets, $\alpha_{\mathbb{P}}(0)=1.20 \pm 0.09$ and $\alpha_{\mathbb{P}}(0)=1.13 \pm 0.04$ respectively, as well as $\alpha_{\mathbb{R}}(0)=0.62 \pm 0.03$ for the Reggeon intercept in case of H1 (there is no need for Reggeons for ZEUS data). In the following, we introduce the Pomeron intercept as a free parameter in the QCD fit.

\section{B. Data sets}

Only data points with virtuality $Q^{2} \geq 3 \mathrm{GeV}^{2}$, diffractive mass at the photon vertex $M_{X} \geq 2 \mathrm{GeV}$ and rapidity $y \leq 0.45$ are included in the fit in order to avoid large higher-twist effects and the region that may be most strongly affected by a non-zero value of the longitudinal over transverse ratio $R$.

The fits include 179 data points for H1 data [2] with 8 parameters (3 for the sea quark density, 3 for the gluon density and 1 for the normalization of the Reggeon contribution and 1 for the Pomeron intercept). Thus, we have 171 degrees of freedom with a $\chi^{2}$ of 203.2 using statistical errors only. In Ref. [8], only 161 data points were included in the QCD fit procedure since an additional cut for $\beta<0.65$ was considered in the analysis. In the new version of the fits, we gain in sensitivity with a larger number of data points, in particular on the gluon density. For example, the third parameter for the gluon density $C_{3}^{(G)}$ was not constrained in Ref. [8] with a value of $0.01 \pm 0.04$ and it is found to be zero in the new analysis. Then, for H1 data, 3 parameters for quarks and 2 parameters for the gluon densties are enough to give a proper description of the cross section measurements through the QCD fit procedure. Also, new results and parameters obtained in Ref. [8] are in perfect agreement for the quarks within the quoted statistical uncertainties.

For ZEUS data [4], the fits include 102 data points with 7 parameters (the parameter corresponding to the normalization of the Reggeon contribution is absent). We have 95 degrees of freedom with a $\chi^{2}$ of 118.4 using statistical errors only. Note that ZEUS measurements are realised for diffractive mass at the proton vertex $M_{Y}<2.3 \mathrm{GeV}$ and squared transfer-momentum $t<1 \mathrm{GeV}^{2}$, whereas $\mathrm{H} 1$ gives diffractive cross sections for $M_{Y}<1.6 \mathrm{GeV}$ and $t<1 \mathrm{GeV}^{2}$. We have converted ZEUS data points to the same $M_{Y}$ range by multiplying ZEUS values by the factor 0.77 [4]. This factor takes into account the conversion from $M_{Y}<2.3 \mathrm{GeV}$ to $M_{Y}<1.6 \mathrm{GeV}$ for the ZEUS measurements, which reflects mainly the fact that the proton-dissociation is reduced for the range of H1 data set. The uncertainty on this number is of course large (of about \pm 0.1 [4]), but we do not take it into account in the following as we do not propose a common fit of $\mathrm{H} 1$ and ZEUS data sets, which would require a relative normalisation error.

On Fig.2, structure function measurements from both data sets are shown. In this last figure, ZEUS data points have been multiplied by the factor 0.77 as explained above, and extrapolated to $\mathrm{H} 1$ bin centers. This extrapolation is done just for the plot, ZEUS data points have been included in the QCD fits at their exact kinematic values.

To get solvable evolution equations, the parton distribution functions must approach zero as $z \rightarrow 1$. This is achieved by introducing in Eqs. (4) the exponential term with a positive value of the parameter $a$. Unless otherwise indicated, in the following fits $a$ is set to 0.01 such that this term only influences the parametrisation in the region $z>0.9$. This term is only present to ensure the convergence and plays a rôle in a domain where we do not include data (for instance, at $\beta=0.65$, its value is equal to 0.97 ).

The functions $z S$ and $z G$ are evolved to higher $Q^{2}$ using the next-to-leading order DGLAP evolution equations (with the code of Ref. [18]), with $\alpha_{S}\left(M_{Z}^{2}\right)=0.118$ (in Ref. [8], a lower value of $\alpha_{S}\left(M_{Z}^{2}\right)$ has been considered). The contribution to $F_{2}^{\mathbb{P}}\left(\beta, Q^{2}\right)$ from charm quarks is calculated in the fixed flavour scheme using the photon-gluon fusion prescription given in Ref. [19] as implemented in Ref. [20]. The contribution from heavier quarks is neglected.

No momentum sum rule has been imposed because of the theoretical uncertainty in specifying the normalization of the Pomeron or Reggeon fluxes.It is also not clear that such a sum rule is appropriate for the parton distributions of a virtually exchanged state. After fitting, however, we observed that the sum rules were fulfilled within $10 \%$ despite being not required in the fits.

\section{Results}

Already from Fig.2 we can get a good idea of the relative gluon densities between H1 and ZEUS data sets. Indeed, we notice a good agreement at $Q^{2}=7.5 \mathrm{GeV}^{2}$ (and $\beta<0.65$ ) whereas both sets of measurements show discrepancies 
at larger $Q^{2}=28 . \mathrm{GeV}^{2}$. ZEUS cross section values are below $\mathrm{H} 1$ for this $Q^{2}$ value, which means that scaling violations in case of ZEUS are lower. Thus, from this basic observation, we expect a lower gluon density in case of ZEUS data compared to H1.

The resulting parton densities of the Pomeron extrapolated from the QCD fit procedure are presented in Fig.1 (extrapolated till $10000 \mathrm{GeV}^{2}$ ) and the parameter values are given in table I.

A few comments are in order :

- We notice that the quark contribution is smaller compared to the gluon one, and much smaller for H1 prediction, confirming the standard results exposed for example in Ref. [8]. On Fig.1, the uncertainties of the parton distributions are not shown but they can be derived from table I, giving typically a $25 \%$ error for the gluon density.

- In the range of the measurements, essentially below $Q^{2}=75 \mathrm{GeV}^{2}$ for $\mathrm{H} 1[2]$ and below $Q^{2}=55 \mathrm{GeV}^{2}$ for ZEUS [4], the agreement between H1 and ZEUS for quark densities is reasonnable.

- Gluon densities for both data sets is quite different, especially at large $\beta$. This last point has been addressed above from the simple observation of scaling violations and is more extensively discussed below.

The result of the fit is presented in Fig.2 together with the experimental values. The fit is plotted only in the $\beta-Q^{2}$ bins considered for the QCD analysis. In this last figure, as mentioned above, ZEUS data points have been converted to the same $M_{Y}<1.6 \mathrm{GeV}$ domain as $\mathrm{H} 1$ (multiplying ZEUS measurements by a multiplicative factor 0.77 as explained above) and extrapolated to $\mathrm{H} 1$ bin centers. However, the QCD-fit procedure and extraction of parton distributions have been done exactly on ZEUS published data points [4]. The extrapolation to H1 bin centers is applied only for the presentation of Fig.2 and we have checked that these conversion factors are small (of the order a few per cent). We see on this figure the good agreement of the QCD fit with the data points, which supports the validity of the description of the Pomeron in terms of partons following perturbative QCD dynamics, even if the accuracy of the gluon determination is still quite poor. This provides a very nice perspective for diffractive analyses of more recent data to be released, where better accuracy will lead to a more precise QCD analysis.

\begin{tabular}{|c|c|c|}
\hline parameters & H1 & ZEUS \\
\hline \hline$\alpha_{\mathbb{P}}$ & $1.19 \pm 0.02$ & $1.13 \pm 0.02$ \\
\hline$C_{1}^{(S)}$ & $0.21 \pm 0.05$ & $0.38 \pm 0.02$ \\
$C_{2}^{(S)}$ & $0.04 \pm 0.02$ & $-0.03 \pm 0.01$ \\
$C_{3}^{(S)}$ & $-0.14 \pm 0.02$ & $-0.11 \pm 0.01$ \\
\hline$C_{1}^{(G)}$ & $0.59 \pm 0.40$ & $0.39 \pm 0.10$ \\
$C_{2}^{(G)}$ & $-0.01 \pm 0.01$ & $-0.36 \pm 0.05$ \\
$C_{3}^{(G)}$ & 0.00 & $0.05 \pm 0.02$ \\
\hline$N_{I R}$ & $14.11 \pm 1.13$ & 0.00 \\
\hline
\end{tabular}

Table I- Pomeron quark and gluon densities parameters. Note that $C_{3}^{(G)}$ is found to be negligeable for H1 data. Also, as mentionned above, there is no Reggeon contribution for ZEUS data. For H1 data, the parameters are close to those of Ref. [8] within the quoted statistical uncertainties, even if we expect some differences as it is explained in the text : for example, more data points are considered and some small changes are done in the QCD fit procedure.

\section{Large $z$ behaviour}

In order to analyze in more detail the large $z$ behaviour of the gluon distribution $z G\left(z, Q^{2}=Q_{0}^{2}\right)$ and give a rough estimate of the systematic error related to our parametrizations, we consider the possibility to change the ansatz (4) by a multiplicative factor $(1-z)^{\nu}$. We shall consider $\nu$ to vary in the interval \pm 1 in order to allow for the still large indetermination of the gluon distribution. If we include this multiplicative factor $(1-z)^{\nu}$ in the QCD fit analysis with statistical errors, we derive a value of $\nu=0.0 \pm 0.6$. Then, the variation in the interval \pm 1 considered here takes also into account a systematic effect of the order of the statistical uncertainty, which is correct. As it is explained more extensively below, the understanding of the large $z$ behaviour is of essential interest for any predictions at the Tevatron or LHC in central dijets production. In particular, a proper determination of the uncertainty in this domain 
of momentum is necessary and the method we propose in this article is a first estimate, that can be propagated to other measurements.

Following Ref. [30], one knows that starting with a given large $z$ input function of the form $(1-z)^{\nu_{0}}$, the effect of leading order DGLAP evolution is to change the end-point exponent by

$$
(1-z)^{\nu_{0}} \rightarrow(1-z)^{\nu_{0}-\frac{16}{33-2 n_{f}}} \log \alpha_{s}\left(Q^{2}\right)
$$

where $n_{f}$ is the number of active flavors, up to a normalization factor.

Hence, let us consider a given solution of the DGLAP evolution equation, such as our structure function $z G\left(z, Q^{2}\right)$, and its large $z$ tail at $Q_{0}^{2}$ parametrized by $(1-z)^{\nu_{0}}$. Then, changing the high $z$ behaviour of the input function (4) by $(1-z)^{ \pm \nu}$ will change the exponent $\nu\left(Q^{2}\right)$ by the same shift, namely

$$
\nu\left(Q^{2}\right) \rightarrow \nu_{0}-\frac{16}{33-2 n_{f}} \log \alpha_{s}\left(Q^{2}\right) \pm \nu \equiv \nu\left(Q^{2}\right) \pm \nu .
$$

Note that the same property is valid for the singlet quark distribution [30].

To be fully consistent with the NLO analysis,we estimated the effect of next-leading DGLAP evolution on the modifications of the high $z$ behaviour due to next-leading effects [31]. We found that the next-leading corrections to the overall high $z$ exponent $1 / \nu\left(Q^{2}\right)$ is of the order of its inverse $1 / \nu\left(Q^{2}\right) \leq 20 \%$ when $\nu\left(Q^{2}\right)$ varies in the kinematical interval in consideration. This correction is negligeable in the conditions of our fits. 

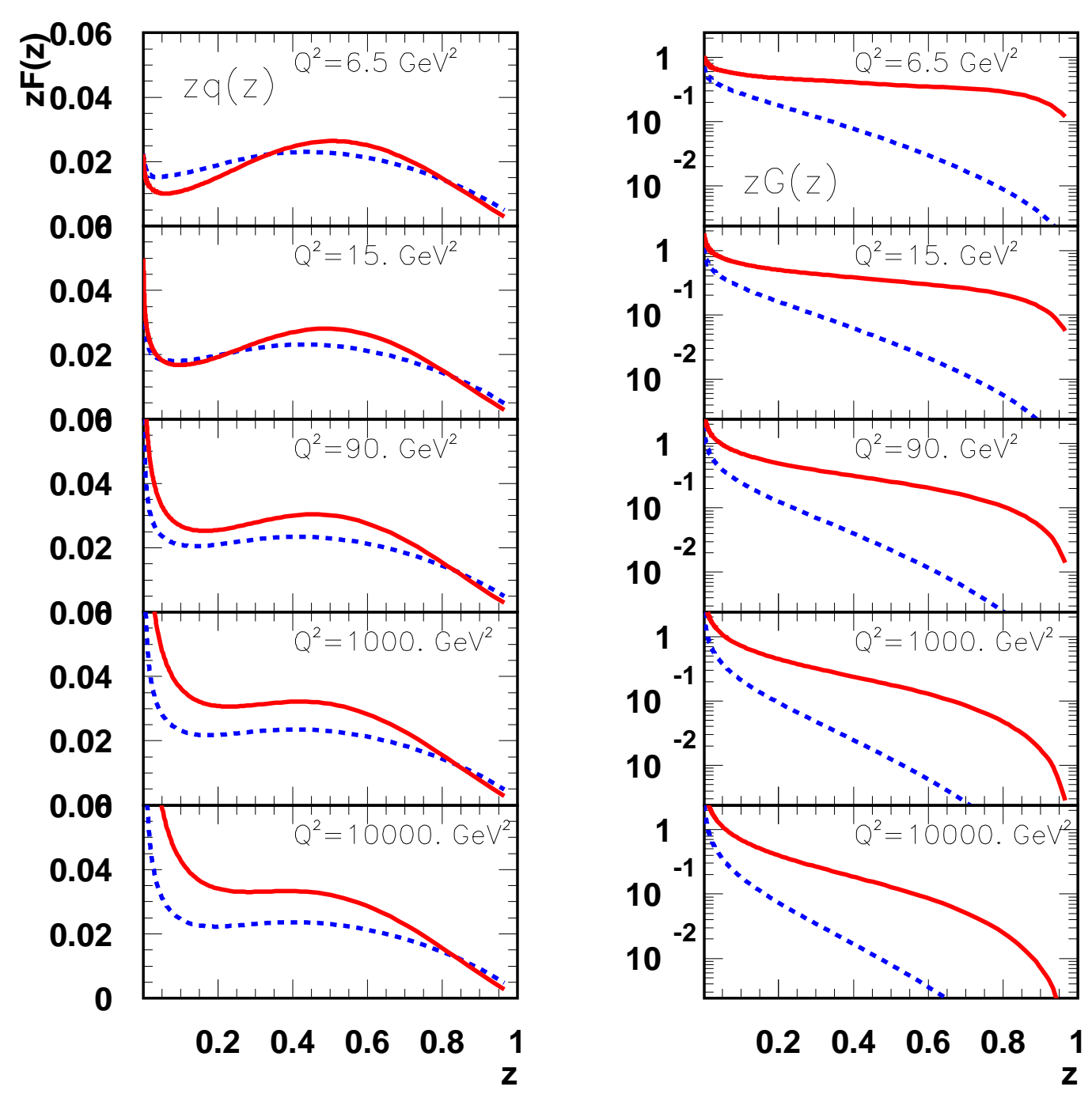

FIG. 1. Quark and Gluon distributions in the Pomeron.(H1 : full line, ZEUS : dotted line) as a function of $z$, the fractional momentum of the Pomeron carried by the struck parton, from the fit on H1 and ZEUS data points with $Q^{2} \geq 3 \mathrm{GeV}^{2}$. The parton densities are normalised to represent $x_{\mathbb{P}}$ times the true parton densities multiplied by the flux factor at $x_{\mathbb{P}}=0.003$. 


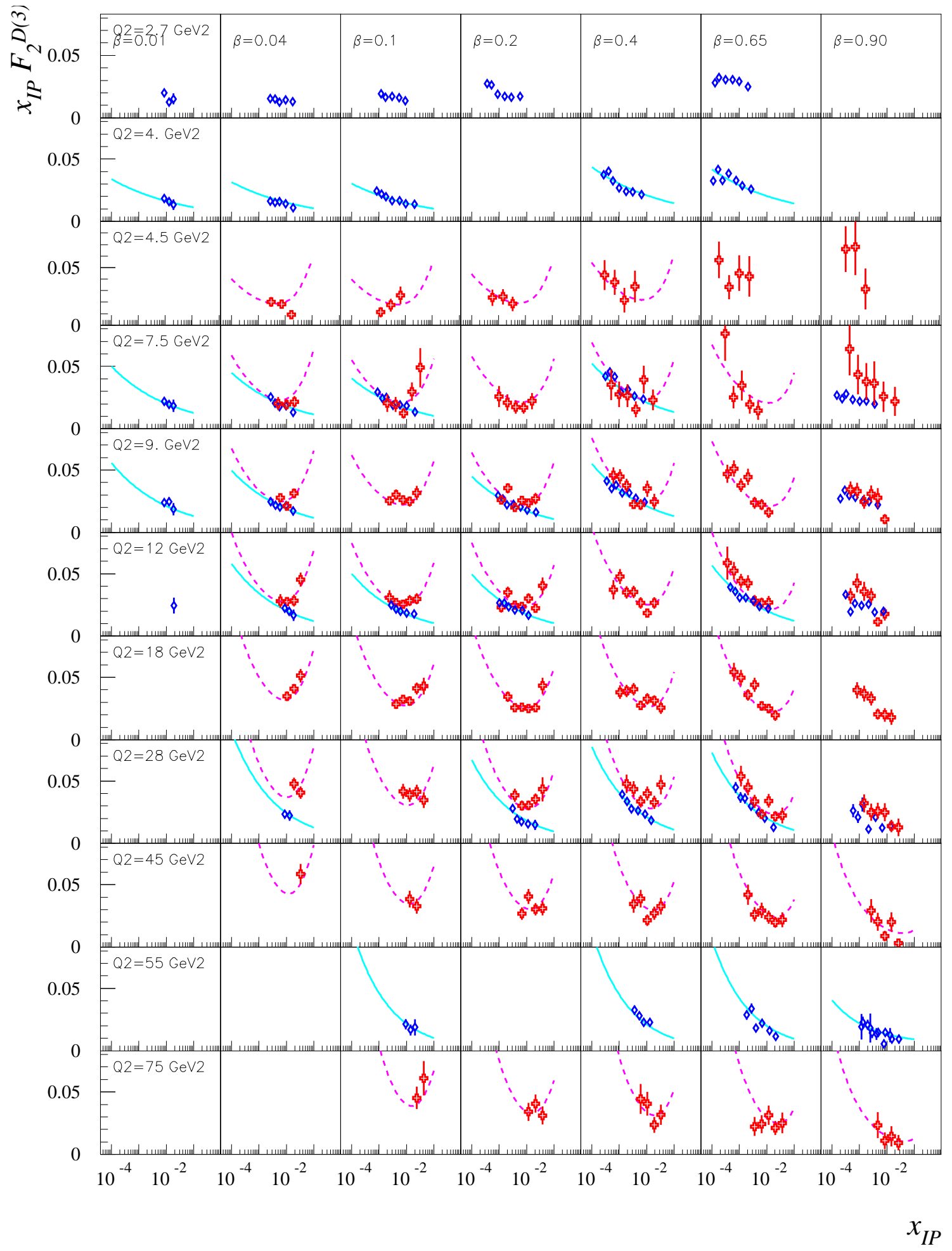

FIG. 2. Comparison of data with the results of the QCD fits. Data points: H1 (dashed line); ZEUS (full line). QCD fits: H1 (crosses); ZEUS (diamonds). The ZEUS data points have been converted to the same $M_{Y}$ range as H1 measurements and moved to the H1 bin centers (see text). 


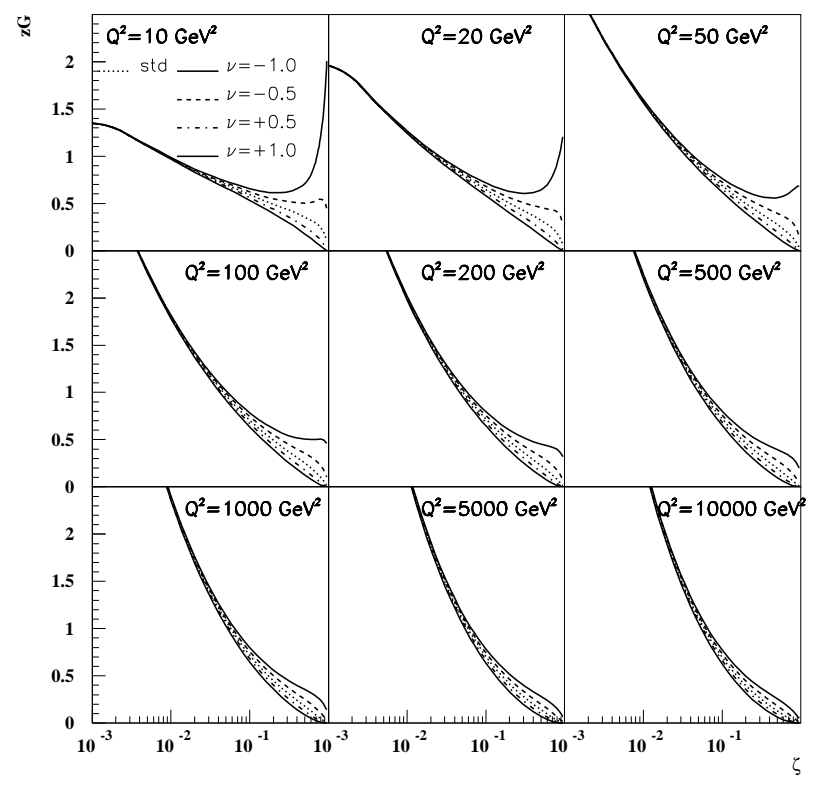

FIG. 3. Gluon distributions of the Pomeron from the fit on $H 1$ data points alone with $Q^{2} \geq 3$ GeV $V^{2}$ The parton densities are normalised to represent $x_{\mathbb{P}}$ times the true parton densities multiplied by the flux factor at $x_{\mathbb{P}}=0.003$. A change by $\nu= \pm .5$ and \pm 1 have been performed (see text).

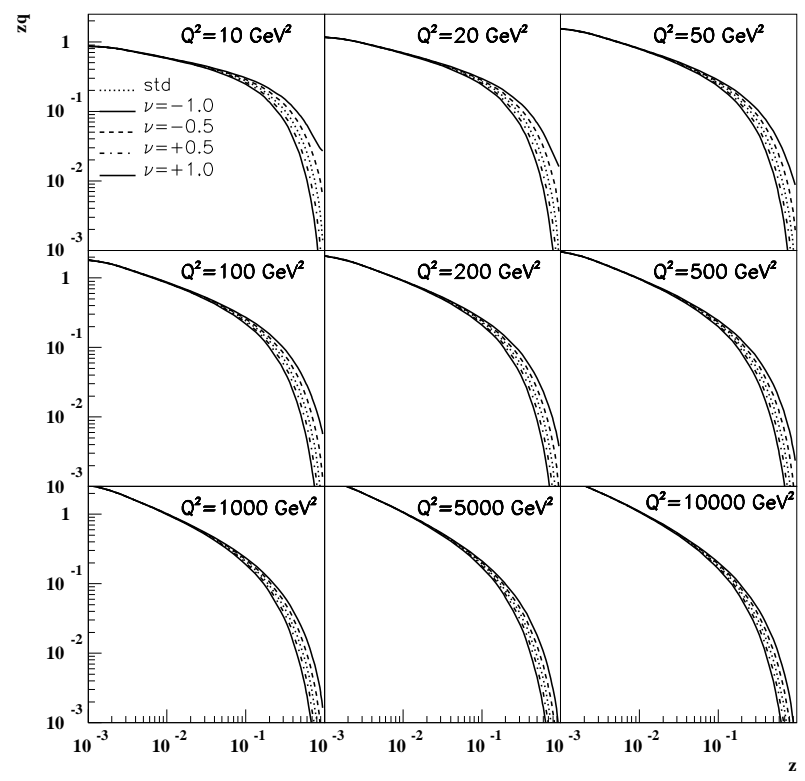

FIG. 4. Gluon distributions of the Pomeron from the fit on ZEUS data points alone with $Q^{2} \geq 3$ GeV $V^{2}$ Same conventions as previous figure. 


\section{EXTRAPOLATION TO TEVATRON AND COMPARISON WITH CDF DATA}

The QCD fits we obtained from HERA data allow us to make direct comparisons for measurements at the Tevatron. It is quite interesting to be able to test directly the factorization breaking between HERA and the Tevatron using the measurements performed at both accelerators. We thus compare the extrapolations of the H1 and ZEUS QCD fits to the recent CDF single diffractive jet cross-section measurement [11]. The result is given in Fig.5. We note a large discrepancy both in shape and normalization between $\mathrm{H} 1$ predictions and CDF data, clearly showing factorization breaking. However, the ZEUS fits are more compatible in normalization with the CDF measurement even if the shape is not described properly. We know that the gluon density from ZEUS is between 2 and 3 times smaller than the one from H1. The predictions for single diffractive production of jets at the Tevatron are thus expected to be different by an sizeable factor 2 to 3 between $\mathrm{H} 1$ and ZEUS since they correspond to single Pomeron exchange. If the large statistical and systematicuncertainties on the gluon density are taken into account (about 50\% for ZEUS, 25\% for H1), ZEUS data are compatible with factorization at low $\beta$.

One should, however, question whether one has the right to extrapolate ZEUS results without introducing the additional Reggeon component. Namely the CDF measurement is in a region in $x_{\mathbb{P}}$ where the Reggeon contribution is important, in contrary to the ZEUS measurement. The error bar on the extrapolation from the fit for ZEUS is thus enhanced due to this uncertainty. Concerning the extrapolation of the H1 fit and the comparison with CDF data, one notices that the CDF data lie primilarly at low values of $\beta$ where there are few H1 data points. Moreover, in this kinematical domain, the $\mathrm{H} 1$ data have the tendency to lie more in the high $x_{\mathbb{P}}$ region where the Reggeon contribution is important and not well constrained by the fit. Thus, the extrapolation to the CDF domain suffers from large uncertainties. A combined fit using CDF data to constrain the low $\beta$ region and the HERA data to constrain the high $\beta$ domain would thus be of great interest.

More precise data from HERA and detailed comparisons with the Tevatron are thus needed to study precisely factorization breaking between both experiments. The discussion of eventual higher twist contributions is clearly also valuable in order to reach conclusions on the shape at larger $\beta$. It is thus important to get an accurate measurement of the gluon density in the Pomeron at low values of $\beta$ from HERA. Furthermore the Forward Proton Detector [29] installed by the D0 collaboration for Run II will be of great help to get a direct measurement of the diffractive structure functions.

\section{POSSIBLE MEASUREMENTS AT THE TEVATRON}

The CDF and D $\varnothing$ experiments at the Tevatron can measure directly the dijet mass fraction, defined as the ratio of the dijet mass to the total diffractive mass measured either in roman pot detectors or in the main $\mathrm{D} \varnothing$ or $\mathrm{CDF}$ detectors.

Let us first notice that we normalised the diffractive dijet cross section using directly the CDF run I measurement previously which takes into account the factorisation breaking between the Tevatron and HERA [8,11]. It has already been mentionned that this measurement is quite sensitive to the Pomeron structure in quark and gluons [8]. We display in Fig.6 the dijet mass fraction for the two different gluon distributions described previously from H1 and ZEUS data after requiring two jets in the central detector with a transverse momentum greater than $25 \mathrm{GeV}$. We indeed see that the difference between the gluon distributions from H1 and ZEUS at HERA can be probed using Tevatron data, provided the survival gap probability (correction which is due to soft interactions) is constant over the full kinematical range. To better show the possibility of such a measurement, we give in Fig.7 the dijet mass fraction when the $\bar{p}$ is tagged in the dipole roman pot detector from the $\mathrm{D} \varnothing$ collaboration. The dipole detectors show a good acceptance for $t$ close to 0 . It would also be possible to require double tagged events (the antiproton in the dipole roman pot detector and the proton in the quadrupole one), but the acceptance of the quadrupole detectors only extends to $t \sim 0.8 \mathrm{GeV}^{2}$ which cuts a lot of events.

On the other hand, it is also possible to probe the high- $\beta$ gluon density in the Pomeron at the Tevatron again through the measurement of the dijet mass fraction (or the total diffractive mass). In Fig8, we givethe dijet mass fraction using the shape of gluon distribution in the Pomeron coming from H1. The sensitivity to the uncertainty on the gluon distribution at high $\beta$ is indicated on that figure by multiplying the gluon distribution by $(1-\beta)^{\nu}$, which enhances or decreases the high $\beta$ gluon distribution. It is quite important to be able to constrain this distribution since it is a direct background to an eventual exclusive signal at high $\beta$. We notice that it is indeed possible to constrain the high- $\beta$ gluon using Tevatron data. At the LHC, it will be possible to constrain better the high- $\beta$ gluon density using higher mass objects, for instance in $t \bar{t}$ inclusive diffractive production. 


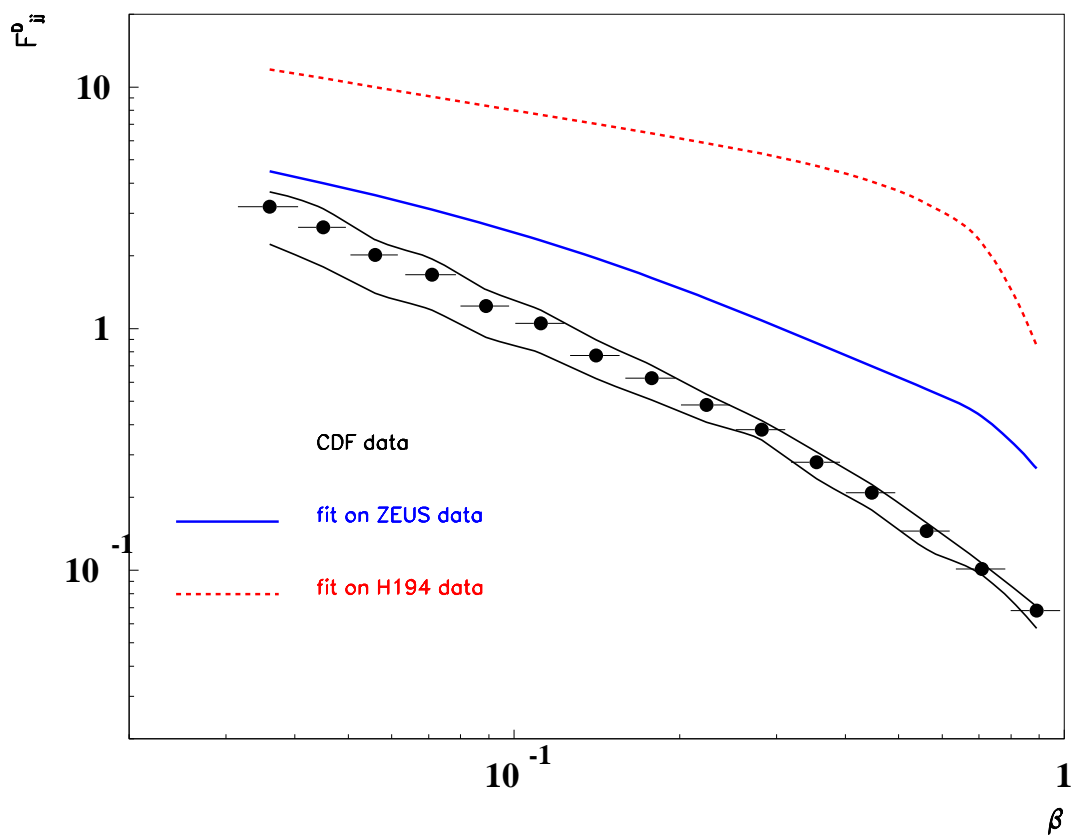

FIG. 5. $\beta$ distributions of CDF data compared with extrapolations from the diffractive parton densities. The partons densities are extracted from $F_{2}^{D(3)}$ measurements by $\mathrm{H} 1$ and by ZEUS collaborations

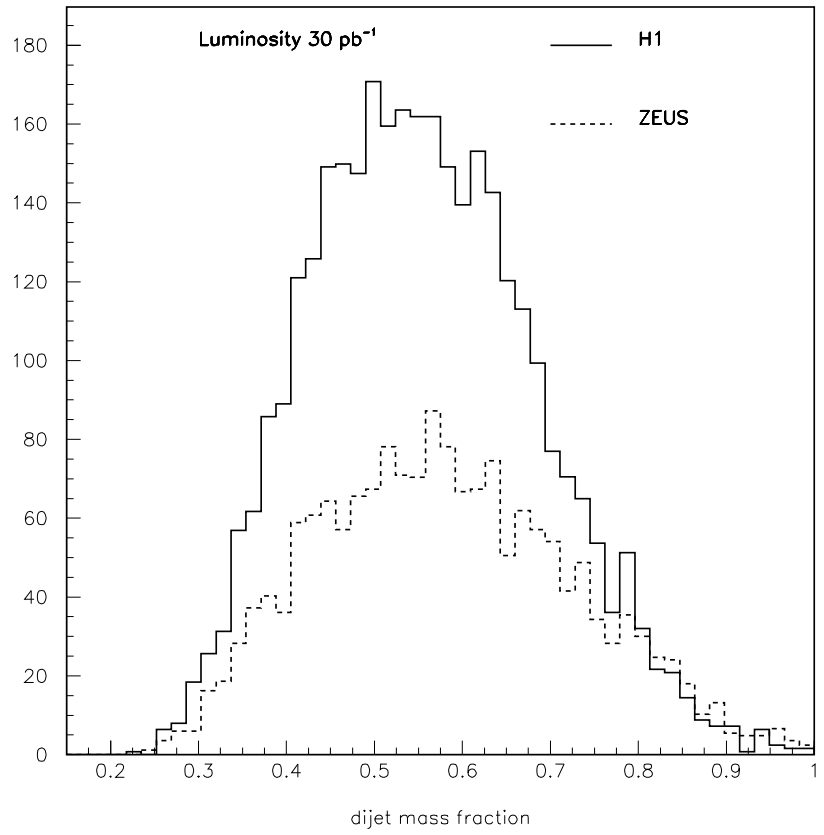

FIG. 6. Results for Dijet mass fraction at generator level. The H1 and ZEUS parton distributions in the Pomeron serve as inputs (see text). 


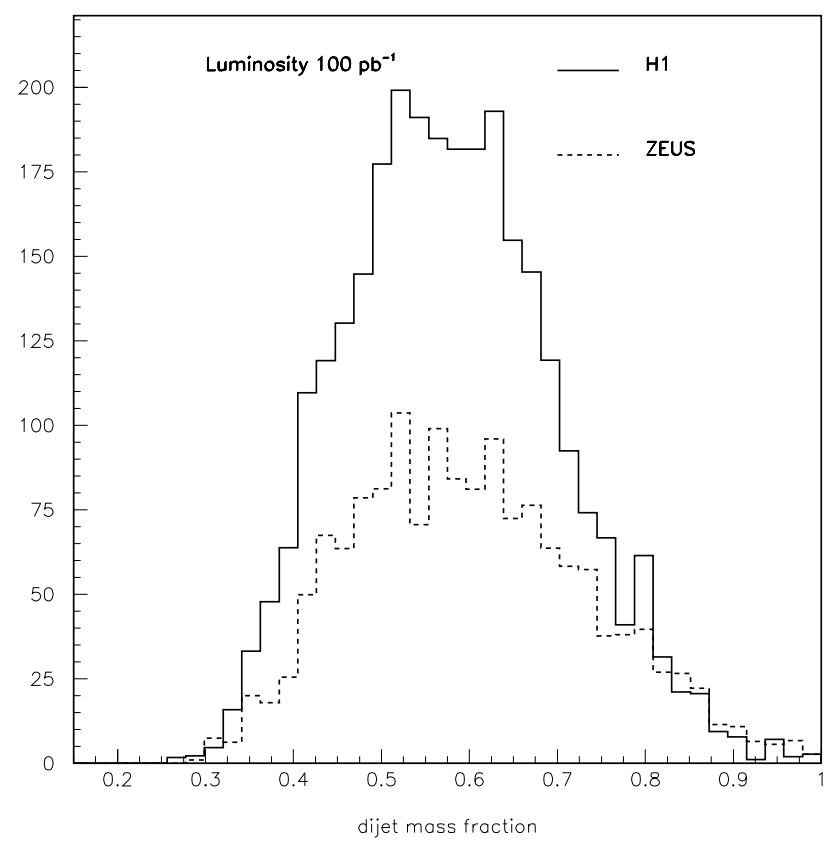

FIG. 7. Results for Dijet mass fraction in the DØ dipole roman pot acceptance at generator level. One considers tagging a $\bar{p}$ (see text). Same convention as previous figure. Note that the CDF results would be similar.

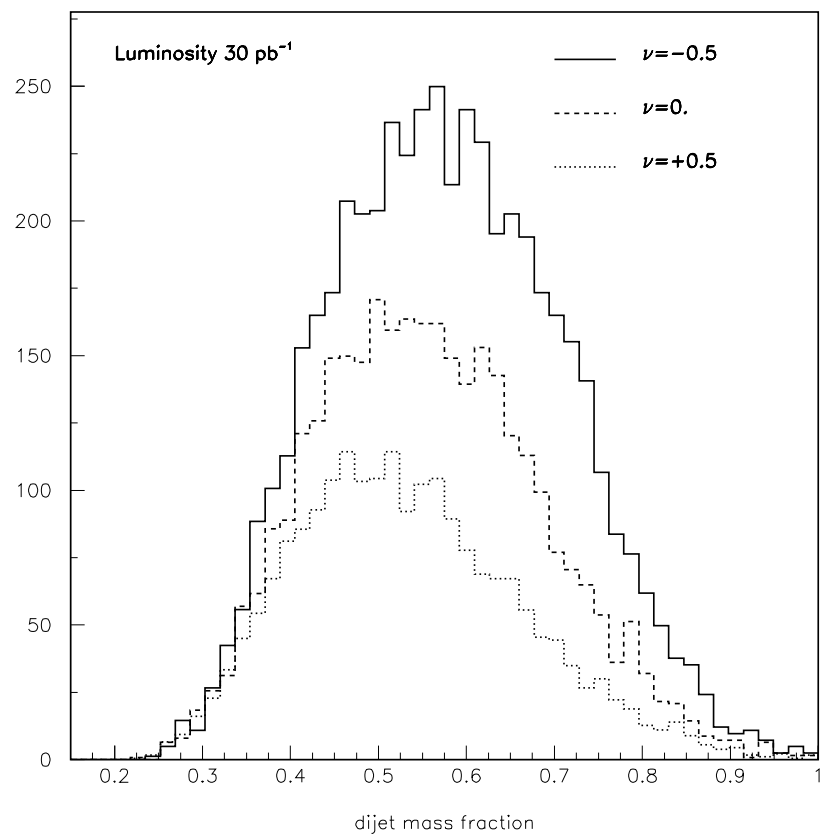

FIG. 8. Results for Dijet mass fraction for the H1 parton distributions as a function of the high- $\beta$ tail. The high- $\beta$ component of the gluon density in the Pomeron is modified ed by a factor $(1-\beta)^{ \pm \nu}$ to show the dependence of the dijet mass fraction on the parameter $\nu$. (see text). 


\section{CONCLUSION}

We have shown that the proton diffractive structure function $F_{2}^{D(3)}$ measured in the H1 and ZEUS experiments at HERA can be well described by a perturbative QCD analysisin which fundamental quark and gluon distributions, evolving according to the next-leading DGLAP equations, are assigned to the leading and sub-leading Regge exchanges.

The gluon distributions have been found to be quite different when extracted from present published data for H1 and ZEUS. An extrapolation to the Tevatron range have been compared with CDF data on single diffraction,leading at this stage to quite different conclusions on factorization breaking between HERA and Tevatron depending on whether H1 (strong violation) or ZEUS (compatibility at low $\beta$ ) fits are used. The discrepancies of the gluon densities between both data sets have also been explained when looking simply at the scaling violations, the QCD fit procedure giving a quantitative estimate of these differences. We have presented a new method to evaluate the uncertainty on the gluon density at large values of $\beta$, which is a key point for any further discussion of the influence of DPDFs at Tevatron or LHC.

In double diffractive dijet production in the central rapidity region, within the double Pomeron formulation, we have found that the Tevatron mass fraction is very sensitive to the high $\beta$ tail of the gluon in the Pomeron. Extrapolation of the fits to very high $Q^{2}$ have been given since they will be relevant for QCD studies at the LHC.

Hence, we have shown that it is possible to constrain the high- $\beta$ gluon using Tevatron data, which may be of decisive interest for any future measurement in central diffractive dijet topologies.

At the LHC, it will be possible to constrain better the high- $\beta$ gluon density using higher mass objects, for instance in $t \bar{t}$ inclusive diffractive production.

We hope that our parametrisations, and the ones easily obtainable exactly with the same technique with forthcoming HERA data, will be useful for the QCD analysis of present and future experiments at the Tevatron and the LHC.

\section{ACKNOWLEDGMENTS}

We want to thank Gregory Soyez for useful discussions on DGLAP equations. 
[1] L.N.Lipatov, Sov. J. Nucl. Phys. 23 (1976) 642; V.S.Fadin, E.A.Kuraev and L.N.Lipatov, Phys. lett. B60 (1975) 50; E.A.Kuraev, L.N.Lipatov and V.S.Fadin, Sov.Phys.JETP 44 (1976) 45, 45 (1977) 199; I.I.Balitsky and L.N.Lipatov, Sov.J.Nucl.Phys. 28 (1978) 822.

[2] H1 Collaboration, Z. Phys. C76 (1997) 613.

[3] ZEUS Collaboration, Eur. Phys. J.C6 (1999) 43.

[4] ZEUS Collaboration, Nucl. Phys. B 713 (2005) 3.

[5] G. Ingelman, P. Schlein, Phys. Lett. B 152 (1985) 256.

[6] J.Collins, Phys.Rev. D57 (1998) 3051, Erratum-ibid. D61 (2000) 019902, F. Hautmann, Z. Kunszt, D. E. Soper "Diffractive parton distributions in light-cone QCD", hep-ph/9911278.

[7] G.Altarelli and G.Parisi, Nucl. Phys. B126 18C (1977) 298. V.N.Gribov and L.N.Lipatov, Sov. Journ. Nucl. Phys. (1972) 438 and 675. Yu.L.Dokshitzer, Sov. Phys. JETP. 46 (1977) 641.

[8] C. Royon, L. Schoeffel, J. Bartels, H. Jung and R. Peschanski, Phys. Rev. D 63 (2001) 074004.

[9] J. Lamouroux, R. Peschanski, C. Royon and L. Schoeffel, Nucl. Phys. B 649 (2003) 312.

[10] H. Jung Comput. Phys. Commun. 86 (1995) 147.

[11] CDF Collaboration, preprint FERMILAB-Pub-/055-E CDF, subm. to Phys.Rev.Lett.

[12] H1 Collab., Z. Phys. C74 (1997) 221.

[13] M. Glück, E. Reya, A. Vogt, Z. Phys. C53 (1992) 651.

[14] J.F. Owens, Phys. Rev. D 30 (1984) 943.

[15] S.Donnachie, P.Landshoff Phys. Lett. B 437 (1998) 408, see also K.Covolan, J.Montanha, K.Goulianos, Phys. Lett. B 389 (1996) 176.

[16] H1 Collaboration, Eur. Phys. J. C6 (1999) 587.

[17] CTEQ QCD evolution code, http://www.phys.psu.edu/cteq/

[18] L. Schoeffel, Nucl.Instrum.Meth. A423 (1999) 439.

[19] M. Glück, E. Hoffmann, E. Reya, Z. Phys. C13 (1982) 119., M. Glück, E. Reya, M. Stratmann, Nucl. Phys. B422 (1994) 37.

[20] H. Jung, Comput. Phys. Commun. 86 (1995) 147.

[21] J.Bartels, J.Ellis, H.Kowalski, M.Wuesthoff, Eur.Phys.J. C7 (1999) 443, J.Bartels, C.Royon, Mod. Phys. Lett. A14 (1999) 1583 .

[22] A.Bialas, R.Peschanski, C.Royon, Phys. Rev. D57 (1998) 6899. S.Munier, R.Peschanski, C.Royon, Nucl. Phys. B534 (1998) 297.

[23] A.H.Mueller and B.Patel, Nucl. Phys. B425 (1994) 471, A.H.Mueller, Nucl. Phys. B437 (1995) 107, A.H.Mueller, Nucl. Phys. B415 (1994) 373; H. Navelet, R. Peschanski, C. Royon. Phys. Lett. B366 (1996) 329; H.Navelet, R.Peschanski, Ch.Royon, S.Wallon, Phys. Lett. B385 (1996) 357.

[24] C.Royon, Contribution to the DIS99 workshop for the H1 Collaboration, Zeuthen (Germany), 19-23 April 1999, hepph/9908216, M.Capua, Contribution to the DIS2000 workshop for the ZEUS Collaboration, Liverpool (U.K.), 25-30 April 2000.

[25] K. Golec-Biernat, M. Wüsthoff, Phys.Rev. D60 (1999) 114023.

[26] J.Cole, Contribution to the DIS2000 workshop for the ZEUS Collaboration, Liverpool (U.K.), 25-30 April 2000.

[27] H1 Collaboration,Phys. Lett. B428 (1998) 206, Eur. Phys. J. C 5 (1998) 3, ZEUS Collaboration, Phys. Lett. B 421 (1998) 368.

[28] H1 Collaboration, Nucl.Phys. B470 (1996) 3.

[29] D0 Collaboration, D0 FPD Proposal, FNAC PAC Meeting October 18, 1997.

[30] C. Lopez and F. J. Yndurain, Nucl. Phys. B 171, 231 (1980).

[31] Nucl. Phys. B 183, 157 (1981). 